\title{
TESTING ANALYTICITY ON ROTATION INVARIANT FAMILIES OF CURVES
}

\author{
JOSIP GLOBEVNIK \\ Dedicated to Professor Ivan Vidav on the occastion of his \\ seventieth birthday, January 17, 1988
}

\begin{abstract}
Let $\Gamma \subset C$ be a piecewise smooth Jordan curve, symmetric with respect to the real axis, which contains the origin in its interior and which is not a circle centered at the origin. Let $\Omega$ be the annulus obtained by rotating $\Gamma$ around the origin. We characterize the curves $\Gamma$ with the property that if $f \in C(\Omega)$ is analytic on $s \Gamma$ for every $s,|s|=1$, then $f$ is analytic in Int $\Omega$.
\end{abstract}

1. Introduction. Throughout the paper we assume that $\Gamma \subset C$ is a piecewise smooth Jordan curve which is symmetric with respect to the real axis, does not contain the origin and is not a circle centered at the origin. We denote by $D$ the bounded domain with boundary $\Gamma$. We denote by $\Omega$ the closed annulus obtained by rotating $\Gamma$ around the origin: $\Omega=\{s z: z \in \Gamma,|s|=1\}$. We denote by $a, b$ the inner and the outer radius of $\Omega$, respectively.

We call the curve $\Gamma$ regular if every continuous function on $\Omega$ which is analytic on each curve $s \Gamma,|s|=1$, is analytic in Int $\Omega$, that is, if $f \in C(\Omega)$ and if

$$
\begin{aligned}
& \text { for each } s \in C,|s|=1 \text {, the function } f \mid(s \Gamma) \text { has a continuous } \\
& \text { extension to } s \bar{D} \text { which is analytic in } s D
\end{aligned}
$$

then $f$ is analytic in Int $\Omega$. We call $\Gamma$ singular if it is not regular.

When studying the conditions which imply the regularity of $\Gamma$ one has to distinguish two cases:

(i) 0 is in the exterior of $\Gamma$, i.e. $0 \in C \backslash \bar{D}$,

(ii) 0 is in the interior of $\Gamma$, i.e. $0 \in D$.

In the first case the situation is simple.

THEOREM 0 [1]. If 0 is in the exterior of $\Gamma$ then $\Gamma$ is regular.

In the present paper we study the second case and from now on we assume that 0 is in the interior of $\Gamma$. Now the situation is more complicated. We illustrate this with two examples.

EXAMPLE 1. Suppose that $\Gamma$ contains an arc of a circle centered at the origin. If $\left|s_{1}\right|=\left|s_{2}\right|=1$ and if $s_{1}$ is close to $s_{2}$ then $b\left(s_{1} D\right) \cap b\left(s_{2} D\right)$ contains an arc. This implies that $\Gamma$ is regular [1].

EXAMPLE 2. Let $\Gamma$ be a circle whose center is different from the origin and which contains the origin in its interior. The function $f(z)=1 / \bar{z}$ shows that $\Gamma$ is singular.

Received by the editors February 1, 1987.

1980 Mathematics Subject Classification (1985 Revision). Primary 30E05, 30E20.

This work was supported in part by the Republic of Slovenia Science Foundation. 
2. A characterization of singular curves. If $f \in C(\Omega), a \leq r \leq b$ and $n \in Z$ define

$$
A_{n}(f, r)=r^{-n} \frac{1}{2 \pi} \int_{0}^{2 \pi} e^{-i n \varphi} f\left(r e^{i \varphi}\right) d \varphi .
$$

Note that if $f$ is analytic in Int $\Omega$ then for each $r, a \leq r \leq b, A_{n}(f, r)$ is equal to the $n$th coefficient in the Laurent series of $f$. Note also that $f$ is analytic in Int $\Omega$ if and only if for each $n \in Z$ the function $r \mapsto A_{n}(f, r)$ is constant on $[a, b][\mathbf{1}]$.

Lemma 1. Suppose that $f \in C(\Omega)$ satisfies (1). Then for every $n \in Z$ the function $z \mapsto z^{n} A_{n}(f,|z|)$ has a continuous extension from $\Gamma$ to $\bar{D}$ which is analytic in $D$.

Note that in [1] the lemma is stated for smooth curves $\Gamma$. However, its proof works equally well for piecewise smooth curves $\Gamma$.

THEOREM 1. $\Gamma$ is singular if and only if there are $n \in N$ and a function $G$, continuous on $\bar{D}$ and analytic in $D$ such that the function $w \mapsto G(w) / w^{n}$ is nonconstant and depends only on $|w|$ on $\Gamma$, that is, if $w_{1}, w_{2} \in \Gamma,\left|w_{1}\right|=\left|w_{2}\right|$, then $G\left(w_{1}\right) / w_{1}^{n}=G\left(w_{2}\right) / w_{2}^{n}$.

ProOF. Suppose that $\Gamma$ is singular. This means that there is an $f \in C(\Omega)$ which satisfies (1) and which is not holomorphic in Int $\Omega$. By Lemma 1 , for each $n \in Z$ there is a continuous function $G_{n}$ on $\bar{D}$, analytic in $D$ and such that $A_{n}(f,|z|)=$ $G_{n}(z) / z^{n}(z \in \Gamma)$. It follows that $A_{n}(f, r)=0(a \leq r \leq b)$ and that $A_{0}(f, r)=$ const $(a \leq r \leq b)[\mathbf{1}]$. Since $f$ is not analytic in Int $\Omega$ there is some $n>0$ such that $r \mapsto A_{n}(f, r)$ is nonconstant. Put $G=G_{n}$. Clearly $G$ has the required properties.

Conversely, suppose that there are $n \in N$ and a continuous function $G$ on $\bar{D}$, analytic in $D$ and such that $w \mapsto G(w) / w^{n}$ is nonconstant and depends only on $|w|$ on $\Gamma$. Define the function $g$ on $\Omega$ by

$$
g\left(|z| e^{i \alpha}\right)=G(z) / z^{n} \quad(z \in \Gamma, 0 \leq \alpha \leq 2 \pi) .
$$

Then $g$ is well defined and continuous on $\Omega$, depends only on $|z|$ and is not a constant. Put $f(z)=z^{n} g(z)(z \in \Omega)$. If $|s|=1$ and $z \in \Gamma$ then $f(s z)=(s z)^{n} g(|z|)=$ $(s z)^{n} G(z) / z^{n}=s^{n} G(z)$. This shows that $f$ satisfies (1). Since $f \in C(\Omega)$ and since $f$ is not analytic in Int $\Omega$ it follows that $\Gamma$ is singular. This completes the proof.

3. Singular curves and symmetry. By our assumption, $\Gamma$ is symmetric with respect to the real axis. A singular curve may have no other lines of symmetry $[\mathbf{1}$, Example 5]. However, once it contains two arcs whose union is symmetric with respect to a line $L$ through 0 then it is symmetric with respect to $L$. This is a consequence of the following

THEOREM 2. Let $\Gamma$ be a singular curve. Suppose that there are an arc $\Lambda \subset \Gamma$ and an $\alpha, 0<\alpha<2 \pi$, such that $e^{i \alpha} \Lambda \subset \Gamma$. Then $\Gamma=e^{i \alpha} \Gamma$ and consequently $\Gamma$ is symmetric with respect to the lines through 0 and $e^{i n \alpha / 2}, n \in N$. In particular $\alpha / \pi$ must be rational.

For a set $E \subset C$ write $E^{*}=\{\bar{\zeta}: \varsigma \in E\}$. To prove Theorem 2 we need the following lemma. 
LEMMA 2 [1]. Let $P \subset C$ be an open set with piecewise smooth boundary. Let $f$ be a continuous function on $\bar{P}$ which is analytic in $P$ and let $g$ be a continuous function on $\bar{P}^{*}$ which is analytic in $P^{*}$. Suppose that $f(w)=g(\bar{w})(w \in b P)$. Then $f$ is a constant.

Proof of TheOREM 2. By Theorem 1 there are $n \in N$ and a function $F$, continuous on $\bar{D}$, analytic in $D$ such that $w \mapsto G(w)=F(w) / w^{n}$ is nonconstant and depends only on $|w|$ on $\Gamma$. So there is a function $\varphi:[a, b] \rightarrow C$ such that $G(w)=\varphi(|w|)(w \in \Gamma)$. Note that $G$ is continuous on $\bar{D} \backslash\{0\}$ and analytic on $D \backslash\{0\}$.

Let $P=D \cap\left(e^{i \alpha} D\right)$. Denote by $P_{1}$ the component of $P$ which contains $e^{i \alpha} \Lambda$ in its boundary. Assume for a moment that $\bar{P}_{1}$ does not contain 0 . Then $G$ is continuous on $\bar{P}_{1}$ and analytic in $P_{1}$. If $w \in\left(b P_{1}\right) \cap \Gamma$ then $G(w)=\varphi(|w|)$. Further, if $w \in e^{i \alpha} \Lambda$ then $e^{-i \alpha} w \in \Lambda$ so $G(w)=\varphi(|w|)=\varphi\left(\left|e^{-i \alpha} w\right|\right)=G\left(e^{-i \alpha} w\right)$. This implies that $G(w)=G\left(e^{-i \alpha} w\right)\left(w \in \bar{P}_{1}\right)$. In particular, if $w \in\left(3 P_{1}\right) \cap\left(e^{i \alpha} \Gamma\right)$ then $G(w)=G\left(e^{-i \alpha} w\right)=\varphi\left(\left|e^{-i \alpha} w\right|\right)=\varphi(|w|)$ so $G(w)=\varphi(|w|)\left(w \in\left(b P_{1}\right) \cap\left(e^{i \alpha} \Gamma\right)\right)$.

Let $Q=D \cap e^{-i \alpha} D$. Denote by $Q_{1}$ the component of $Q$ which contains $\left(e^{i \alpha} \Lambda\right)^{*}=$ $e^{-i \alpha} \Lambda^{*}$ in its boundary. Note that $Q_{1}^{*}=P_{1}$. So $\bar{Q}_{1}$ does not contain 0 and consequently $G$ is continuous on $\bar{Q}_{1}$ and analytic in $Q_{1}$. If $w \in\left(b Q_{1}\right) \cap \Gamma$ then $G(w)=\varphi(|w|)$. Further, if $w \in\left(e^{i \alpha} \Lambda\right)^{*}$ then $e^{i \alpha} w \in \Lambda^{*}$ so $G(w)=\varphi(|w|)=$ $\varphi\left(\left|e^{-i \alpha} w\right|\right)=G\left(e^{i \alpha} w\right)$. This implies that $G(w)=G\left(e^{i \alpha} w\right)\left(w \in \bar{Q}_{1}\right)$. In particular, if $w \in\left(b Q_{1}\right) \cap\left(e^{-i \alpha} \Gamma\right)$ then $G(w)=G\left(e^{i \alpha} w\right)=\varphi\left(\left|e^{i \alpha} w\right|\right)=\varphi(|w|)$ so $G(w)=$ $\varphi(|w|)\left(w \in\left(b Q_{1}\right) \cap\left(e^{-i \alpha} \Gamma\right)\right)$.

We have proved that $G$ is continuous on $\bar{P}_{1}$, analytic in $P_{1}$, continuous on $\bar{P}_{1}^{*}$, analytic in $P_{1}^{*}$ and satisfies $G(w)=\varphi(|w|)\left(w \in b P_{1}, w \in b P_{1}^{*}\right)$. So $G(w)=G(\bar{w})$ $\left(w \in b P_{1}\right)$. By Lemma 2 it follows that $G$ is constant on $\bar{P}_{1}$, a contradiction.

Thus we proved that $0 \in \bar{P}_{1}$. Since $P$ contains a neighborhood of 0 it follows that $0 \in P_{1}$.

Let $B=D \backslash \bar{P}_{1}$ and assume that $B$ is not empty. If we repeat the above argument we see that $G(w)=\varphi(|w|)\left(w \in b P_{1}\right)$. Further, since $G(w)=\varphi(|w|)(w \in \Gamma=b D)$ it follows that $G$ is continuous on $\bar{B}$, analytic in $B$ and satisfies $G(w)=\varphi(|w|)$ $(w \in b B)$. In the same way, considering $B^{*}=D \backslash \bar{Q}_{1}$ instead of $B$ we prove that $G$ is continuous on $\bar{B}^{*}$, analytic in $B^{*}$ and satisfies $G(w)=\varphi(|w|)\left(w \in b B^{*}\right)$. So $G(\bar{w})=G(w)(w \in b B)$ and by Lemma $2 G$ is a constant, a contradiction. Consequently $B=\varnothing$ so $D \subset \bar{P}_{1} \subset \bar{D} \subset e^{i \alpha} D$ which implies that $D=e^{i \alpha} D$ and $\Gamma=e^{i \alpha} \Gamma$. Further, since $e^{i n \alpha} D=D(n \in N)$ and since $D^{*}=D$ it follows that $\left(e^{-i n \alpha / 2} D\right)^{*}=e^{i n \alpha / 2} D=e^{-i n \alpha / 2} D$ which proves that $\Gamma$ is symmetric with respect to the lines through 0 and $e^{i n \alpha / 2}, n \in N$. Since $\Gamma$ is not a circle centered at 0 it follows that $\alpha / \pi$ must be rational. This completes the proof.

COROLlaRY 1. Let $\Gamma$ be a singular curve. Suppose that $0<\beta<\pi$ and that $\Gamma$ contains two arcs whose union is symmetric with respect to the line $L$ through 0 and $e^{i \beta}$. Then $\Gamma$ is symmetric with respect to $L$. In particular, $\beta / \pi$ must be rational.

PROOF. By the assumption there are arcs $\Lambda_{1}, \Lambda_{2}$ such that $\left(e^{-i \beta} \Lambda_{1}\right)^{*}=e^{-i \beta} \Lambda_{2}$ which implies that $e^{2 i \beta} \Lambda_{1}^{*}=\Lambda_{2}$. Since $\Gamma^{*}=\Gamma$ it follows that $\Lambda_{1}^{*} \subset \Gamma$ and Theorem 2 implies that $\Gamma$ is symmetric with respect to $L$. This completes the proof. 
4. Two examples. We denote by $\Delta$ the open unit disc in $C$.

PROPOSITION 1. Let $\Gamma$ be a triangle. Then $\Gamma$ is singular if and only if $\Gamma$ is an equilateral triangle centered at the origin.

Recall that we are assuming that $\Gamma=\Gamma^{*}$.

Proof. Suppose that $\Gamma$ is singular. By Corollary 1 the lines through 0 which are perpendicular to the sides of $\Gamma$ are the lines of symmetry for $\Gamma$ which proves that $\Gamma$ is an equilateral triangle centered at 0 . Conversely, suppose that $\Gamma$ is an equilateral triangle centered at 0 . Let $\Psi: D \rightarrow \Delta$ be the conformal map which satisfies $\Psi(0)=0, \Psi^{\prime}(0)>0$. Define

$$
G(w) / w^{3}=\Psi(w)^{3}+1 / \Psi(w)^{3} .
$$

Then $G$ is continuous on $\bar{D}$, analytic in $D$ and it is easy to see that $G(w) / w^{3}$ depends only on $|w|$ on $\Gamma$. By Theorem $1 \Gamma$ is singular. This completes the proof.

PROPOSITION 2. Let $\Gamma$ be a rectangle. Then $\Gamma$ is singular if and only if $\Gamma$ is a square centered at the origin.

ProOF. Suppose that $\Gamma$ is a square centered at the origin. Let $\Psi: D \rightarrow \Delta$ be the conformal map which satisfies $\Psi(0)=0, \Psi^{\prime}(0)>0$. Then

$$
G(w) / w^{4}=\Psi(w)^{4}+1 / \Psi(w)^{4}
$$

depends only on $|w|$ on $\Gamma$. In the same way as above, Theorem 1 implies that $\Gamma$ is singular.

Conversely, assume that $\Gamma$ is singular. Corollary 1 implies that $\Gamma$ is symmetric with respect to the imaginary axis. With no loss of generality assume that $a+i$, $a-i,-a+i,-a-i$ are the vertices of $\Gamma$ where $a>0$. We have to prove that $a=1$.

It suffices to prove the following: Suppose that there is a nonconstant function $G$, continuous on $\bar{D} \backslash\{0\}$ and analytic in $D \backslash\{0\}$ which depends only on $|w|$ on $\Gamma$. Then $a=1$. Let $G$ be as above. With no loss of generality assume that $G(a+i)=0$. Since $G(w)$ depends only on $|w|$ on $\Gamma$ it follows that we can extend $G$ to the rectangle $Q$ with vertices $3 a-i, 3 a+3 i,-a-i,-a+3 i$ by

$$
\left.\begin{array}{l}
G(2 i+w)=G(w), \\
G(2 a+w)=G(w), \\
G(2 a+2 i+w)=G(w)
\end{array}\right\} \quad(w \in \bar{D} \backslash\{0\})
$$

to get a continuous function on $\bar{Q} \backslash\{0,2 a, 2 i, 2 a+2 i\}$. In particular, $G$ is analytic in a neighborhood of $a+i$. Put $f(w)=G(w-(a+i))$. Then $f$ is analytic in an open disc $U$ centered at 0 . By the properties of $G$ we have $f(w)=f(-w)(w \in U \cap R)$ so $f(w)=f(-w)(w \in U)$. Further, whenever $z \in R_{-}$and $w \in i R_{-}$have the same distance from $-a-i$ we have $f(w)=f(z)$.

Put $w(z)=i\left(\left(z^{2}+2 a z+1\right)^{1 / 2}-1\right)$ where $1^{1 / 2}=1$. Passing to a smaller $U$ if necessary we may assume that $w$ is analytic in $U$. We have

$$
(z+a)^{2}+1=(w(z) / i+1)^{2}+a^{2} \quad(z \in U)
$$

which means that if $z \in U \cap R_{-}$then $w \in i R_{-}$and $w$ and $z$ have the same distance from $-a-i$. 
There is a disc $U^{\prime}$ centered at 0 such that $w\left(U^{\prime}\right) \subset U$. For every $z \in U^{\prime} \cap R_{-}$we have $f(w(z))=f(z)$ which implies that

$$
f(w(z))=f(z) \quad\left(z \in U^{\prime}\right) .
$$

Recall that $f(0)=0$. Since $f$ is not a constant there is some $k \in N$ such that $f^{\prime}(0)=\cdots=f^{(k)}(0)=0, f^{(k+1)}(0) \neq 0$. By (2) we have

$$
\begin{aligned}
f^{(k+1)}(z)= & f^{(k+1)}(w(z)) w^{\prime}(z)^{k+1} \\
& + \text { terms containing } f^{(k)}(w(z)), \ldots, f^{\prime}(w(z)) \text { as factors, }
\end{aligned}
$$

so $f^{(k+1)}(0)=f^{(k+1)}(0) w^{\prime}(0)^{k+1}$ which implies that $w^{\prime}(0)^{k+1}=1$. On the other hand, $w^{\prime}(0)=i a$ by the definition of $w(z)$. Consequently $a=1$. This completes the proof.

REMARK. There is no similar result when $\Gamma$ is a pentagon. There are pentagons $\Gamma$ which are singular and which are not equilateral. To see this observe that there are pentagons of the same form as [1, Example 5].

5. Functions analytic on $s \Gamma,|s|=1$. We now proceed to obtain a more detailed description of singular curves. The first step in this direction is a characterization of functions $f$ which satisfy (1).

From now on we denote by $\Phi$ the conformal map from $\Delta$ to $D$ which satisfies $\Phi(0)=0, \Phi^{\prime}(0)>0$.

THEOREM 3. A function $f \in C(\Omega)$ satisfies (1) if and only if the following two conditions are satisfied:

(i) for each $n<0, A_{n}(f, r)=0(a \leq r \leq b)$,

(ii) for each $n \geq 0$ there is a polynomial $P_{n}$ satisfying $P_{n}(1 / \varsigma)=\varsigma^{-2 n} P_{n}(\varsigma)$ $(s \in C)$ such that

$$
A_{n}(f,|\Phi(\varsigma)|)=\varsigma^{-n} P_{n}(\varsigma) \quad(\varsigma \in b \Delta) .
$$

REMARK. Note that the degree of $P_{n}$ is at most $2 n$. Note also that $\varsigma^{-n} P_{n}(\varsigma)$ has the form $b_{n 0}+b_{n 1}(\varsigma+1 / \varsigma)+\cdots+b_{n n}\left(\varsigma^{n}+1 / \varsigma^{n}\right)$ so that (ii) is equivalent to

(ii') for each $n \geq 0$ there are $a_{n 0}, a_{n 1}, \ldots, a_{n n}$ such that

$$
A_{n}\left(f,\left|\Phi\left(e^{i \varphi}\right)\right|\right)=a_{n 0}+a_{n 1} \cos \varphi+\cdots+a_{n n} \cos n \varphi .
$$

PROOF. The only if part. Observe first that by the symmetry of $\Gamma$ with respect to the real axis we have $\Phi(\bar{\zeta})=\overline{\Phi(\varsigma)}(\varsigma \in b \Delta)$.

Assume that $f \in C(\Omega)$ satisfies (1). By Lemma 1 for every $n \in Z$ the function $z \mapsto z^{n} A_{n}(|z|)$ has a continuous extension from $\Gamma$ to $\bar{D}$ which is analytic in $D$. It follows that for each $n \in Z$ there is a function $F_{n}$, continuous on $\bar{\Delta}$, analytic in $\Delta$, such that

$$
\Phi(\varsigma)^{n} A_{n}(f,|\Phi(\varsigma)|)=F_{n}(\varsigma) \quad(\varsigma \in b \Delta) .
$$

If $n<0$ it follows that $\varsigma \mapsto A_{n}(f,|\Phi(\varsigma)|)$ is the boundary function of a function $G$, continuous on $\bar{\Delta}$, analytic in $\Delta$, which has a zero at $\varsigma=0$. Since $G(\varsigma)=G(\bar{\zeta})$ $(\varsigma \in b \Delta)$ it follows that $G=0$ which proves (i).

Let $n \geq 0$. We have $\Phi(\varsigma)=\zeta \Psi(\zeta)(\zeta \in \bar{\Delta})$ where both $\Psi$ and $1 / \Psi$ are continuous on $\bar{\Delta}$ and analytic in $\Delta$. By (3) we have

$$
F_{n}(\varsigma) / \Psi(\varsigma)^{n}=\varsigma^{2 n} F_{n}(1 / \varsigma) / \Psi(1 / \varsigma)^{n} \quad(\varsigma \in b \Delta)
$$


which implies that

$$
\varsigma \mapsto \begin{cases}F_{n}(\varsigma) / \Psi(\varsigma)^{n} & (\zeta \in \bar{\Delta}), \\ F_{n}(1 / \zeta) \cdot \varsigma^{2 n} / \Psi(1 / \zeta)^{n} & (\varsigma \in C \backslash \Delta),\end{cases}
$$

is an entire function of polynomial growth at infinity so it is a polynomial that we denote by $P_{n}$. By (4) we have $P_{n}(\varsigma)=\varsigma^{2 n} P_{n}(1 / \varsigma)(\varsigma \in C)$. Further, by (3), $A_{n}(f,|\Phi(\varsigma)|)=F_{n}(\varsigma) / \Phi(\varsigma)^{n}=\varsigma^{-n} P_{n}(\varsigma)(\varsigma \in b \Delta)$ which proves (ii). This completes the proof of the only if part.

The if part. Suppose that $f \in C(\Omega)$ satisfies (i) and (ii). We first show that for each $n \in Z$ and for each $s,|s|=1$, the function $z \mapsto z^{n} A_{n}(f,|z|)$ has a continuous extension from $s \Gamma$ to $s \bar{D}$ which is analytic in $s D$, that is, $\varsigma \mapsto(s \Phi(\zeta))^{n} A_{n}(f,|\Phi(\zeta)|)$ is the boundary function of a function continuous on $\bar{\Delta}$, analytic in $\Delta$. If $n<0$ this is so since $A_{n}(r)=0(a \leq r \leq b)$. If $n>0$ then

$$
(s \Phi(\varsigma))^{n} A_{n}(f,|\Phi(\varsigma)|)=s^{n} \Psi(\varsigma)^{n} \varsigma^{n} \zeta^{-n} P_{n}(\varsigma)=s^{n} \Psi(\varsigma)^{n} P_{n}(\varsigma)
$$

which, on $b \Delta$, is the boundary function of $s^{n} \Psi P_{n}$ which is continuous on $\bar{\Delta}$ and analytic in $\Delta$. Let

For each $r, a \leq r \leq b, \sum_{-\infty}^{\infty} A_{n}(f, r) r^{n} e^{i n \varphi}$ is the Fourier series of $\varphi \mapsto f\left(r e^{i \varphi}\right)$.

$\sigma_{m}\left(f, r, e^{i \varphi}\right)=m^{-1}\left(A_{0}(f, r)+\sum_{-1}^{1} A_{k}(f, r) r^{k} e^{i k \varphi}+\cdots+\sum_{-(m-1)}^{m-1} A_{k}(f, r) r^{k} e^{i k \varphi}\right)$

be its $m$ th Cezàro mean. By the uniform continuity of $f$ on $\Omega$ the family $\{\theta \mapsto$ $\left.f\left(r e^{i \theta}\right): a \leq r \leq b\right\}$ is uniformly equicontinuous on $[0,2 \pi]$. The usual proof of Féjer's theorem [2] applied to $\sum_{-\infty}^{\infty} A_{n}(f, r) r^{n} e^{i n \varphi}$ shows that $\sigma_{m}\left(f, r, e^{i \varphi}\right)$ converges to $f\left(r e^{i \varphi}\right)$ uniformly for $r$ and $\varphi, a \leq r \leq b, 0 \leq \varphi \leq 2 \pi$. Consequently, on $\Omega, f(z)$ is the uniform limit of the sequence

$$
f_{m}(z)=m^{-1}\left(A_{0}(f,|z|)+\sum_{-1}^{1} A_{k}(f,|z|) z^{k}+\cdots+\sum_{-(m-1)}^{m-1} A_{k}(f,|z|) z^{k}\right) .
$$

By the preceding discussion each $f_{m}$ satisfies (1) so the same holds for $f$. This completes the proof of Theorem 3 .

\section{Another characterization of singular curves.}

THEOREM 4. Let $\Gamma$ and $\Phi$ be as in Theorem 3. $\Gamma$ is singular if and only if there are $n>0$ and real numbers $a_{1}, a_{2}, \ldots, a_{n}$, not all equal to zero, and a function $F$ on $[a, b]$ such that

$$
F(|\Phi(\varsigma)|)=a_{1}(\varsigma+1 / \varsigma)+a_{2}\left(\varsigma^{2}+1 / \varsigma^{2}\right)+\cdots+a_{n}\left(\varsigma^{n}+1 / \varsigma^{n}\right) \quad(\varsigma \in b \Delta) \text {. }
$$

REMARK. (5) should be understood as an incidence relation, that is, if

$$
h(\varsigma)=a_{1}(\varsigma+1 / \varsigma)+\cdots+a_{n}\left(\varsigma^{n}+1 / \varsigma^{n}\right)
$$

then $h\left(\varsigma_{1}\right)=h\left(\varsigma_{2}\right)$ whenever $\varsigma_{1}, \varsigma_{2} \in b \Delta$ and $\left|\Phi\left(\varsigma_{1}\right)\right|=\left|\Phi\left(\varsigma_{2}\right)\right|$. Note also that $h\left(e^{i \varphi}\right)$ is a trigonometric polynomial

$$
h\left(e^{i \varphi}\right)=c_{1} \cos \varphi+\cdots+c_{n} \cos n \varphi .
$$


PROOF. The only if part. Suppose that $\Gamma$ is singular. This means that there is an $f \in C(\Omega)$ which satisfies (1) and which is not holomorphic in Int $\Omega$. It follows that there is some $n \in Z$ such that $r \mapsto A_{n}(f, r)$ is nonconstant on $[a, b][\mathbf{1}]$. By Theorem 3 it follows that there is some $n>0$ such that

$$
A_{n}(f,|\Phi(\varsigma)|)=\varsigma^{-n} P_{n}(\varsigma) \quad(\varsigma \in b \Delta)
$$

where $\varsigma \mapsto \varsigma^{-n} P_{n}(\varsigma)$ is not a constant and $P_{n}$ is a polynomial satisfying $P_{n}(1 / \varsigma)=$ $\varsigma^{-2 n} P_{n}(\varsigma)(\varsigma \in C)$, that is,

$$
A_{n}(f,|\Phi(\varsigma)|)=b_{0}+b_{1}(\varsigma+1 / \varsigma)+\cdots+b_{n}\left(\varsigma^{n}+1 / \varsigma^{n}\right) \quad(\varsigma \in b \Delta)
$$

where at least one of $b_{1}, \ldots, b_{n}$ is different from zero. If all nonzero $b_{k}$ are pure imaginary let

$$
F(|\Phi(\varsigma)|)=i^{-1}\left(A_{n}(f,|\Phi(\varsigma)|)-b_{0}\right) \quad(\varsigma \in b \Delta),
$$

and otherwise let

$$
F(|\Phi(\varsigma)|)=\operatorname{Re}\left(A_{n}(f,|\Phi(\varsigma)|)-b_{0}\right) \quad(\varsigma \in b \Delta) .
$$

In either case $F(|\Phi(\varsigma)|)$ is of the form (5). This completes the proof of the only if part.

The if part. Suppose that there are $n>0$ and real numbers $a_{1}, \ldots, a_{n}$, not all equal to zero, and a function $F$ on $[a, b]$ such that (5) holds. Let $h$ be as in the Remark. By $(5), F(|w|)=h\left(\Phi^{-1}(w)\right)(w \in \Gamma)$. Suppose for a moment that $F$ is not continuous on $[a, b]$. This implies that there are $r, a \leq r \leq b$, and a sequence $w_{n} \in \Gamma,\left|w_{n}\right| \rightarrow r$, such that $\left|F\left(\left|w_{n}\right|\right)-F(r)\right| \geq \delta>0(n \in N)$. By the compactness of $\Gamma$ we may, passing to a subsequence if necessary, assume that $w_{n} \rightarrow w \in \Gamma$. It follows that $\left|\left(h \circ \Phi^{-1}\right)\left(w_{n}\right)-\left(h \circ \Phi^{-1}\right)(w)\right| \geq \delta(n \in N)$, a contradiction. This proves that $F$ is continuous on $[a, b]$. Define

$$
f(z)=z^{n} F(|z|) \quad(z \in \Omega) .
$$

Clearly $f$ is continuous on $\Omega$. Let $s \in b \Delta$. We have $f(s \Phi(\varsigma))=s^{n} \Phi(\varsigma)^{n} h(\varsigma)$ $(\varsigma \in b \Delta)$. Since $h$ has a pole of at most order $n$ at the origin and $\Phi$ has a zero at the origin it follows that $f \mid(s \Gamma)$ has a continuous extension to $s \bar{D}$ which is analytic in $s D$. It follows that $f$ satisfies (1). Since $F$ is not a constant it follows that $f$ is not analytic in Int $\Omega$. This proves that $\Gamma$ is singular. The proof is complete.

\section{Properties of singular curves.}

PROPOSITION 3. Suppose that $\Gamma$ intersects a circle centered at the origin in an infinite set of points. Then $\Gamma$ is regular.

Proof. Suppose that $\Gamma$ is singular. By Theorem 4 there are $n>0$ and numbers $a_{1}, \ldots, a_{n}$, not all equal to zero, and a function $F$ on $[a, b]$ such that $F(|\Phi(\varsigma)|)=h(\varsigma)$ $(\varsigma \in b \Delta)$ where $h(\varsigma)=a_{1}(\varsigma+1 / \varsigma)+\cdots+a_{n}\left(\varsigma^{n}+1 / \varsigma^{n}\right)$. By our assumption $h$ has the same value at an infinite sequence of points on $b \Delta$, a contradiction. This completes the proof.

PROPOSITION 4. Let $\Gamma$ be singular. Then $|w|$ has only a finite number of local maxima or minima on $\Gamma$, that is, $\varphi \mapsto\left|\Phi\left(e^{i \varphi}\right)\right|$ has only a finite number of local maxima or minima on $(0, \pi)$.

ProOF. By Theorem 4 there is a trigonometric polynomial $h(\varphi)=b_{1} \cos \varphi+$ $\cdots+b_{n} \cos n \varphi$, not identically zero, such that $\left|\Phi\left(e^{i \varphi}\right)\right|=\left|\Phi\left(e^{i \psi}\right)\right|$ implies that 
$h(\varphi)=h(\psi)$. Suppose now that $\varphi \mapsto\left|\Phi\left(e^{i \varphi}\right)\right|$ has a local maximum at $\varphi_{0}, 0<$ $\varphi_{0}<\pi$. By Proposition 3 there is a neighborhood $U$ of $\varphi_{0}$ such that $\left|\Phi\left(e^{i \varphi}\right)\right|<$ $\left|\Phi\left(e^{i \varphi_{0}}\right)\right|\left(\varphi \in U \backslash\left\{\varphi_{0}\right\}\right)$. Continuity of $\Phi$ implies that there are sequences $\varphi_{n}^{\prime} / \varphi_{0}$, $\varphi_{n}^{\prime \prime} \searrow \varphi_{0}$ such that $\left|\Phi\left(e^{i \varphi_{n}^{\prime}}\right)\right|=\left|\Phi\left(e^{i \varphi_{n}^{\prime \prime}}\right)\right|(n \in N)$ and consequently $h\left(\varphi_{n}^{\prime}\right)=h\left(\varphi_{n}^{\prime \prime}\right)$ $(n \in N)$. It follows that $h^{\prime}\left(\varphi_{0}\right)=0$. Since $h^{\prime}$ has only finitely many zeros on $(0, \pi)$ it follows that $\varphi \mapsto\left|\Phi\left(e^{i \varphi}\right)\right|$ has only finitely many local maxima or minima on $(0, \pi)$. This completes the proof.

PROPOSITION 5. Let $\Gamma$ be such that $\theta \mapsto\left|\Phi\left(e^{i \theta}\right)\right|$ has only a finite number of local extrema on $(0, \pi)$. Moreover, assume that whenever $\varphi$ is the point of local extremum then $\Gamma$ is symmetric with respect to the line through 0 and $\Phi\left(e^{i \varphi}\right)$. Then $\Gamma$ is singular.

ProOF. Let $\varphi_{i}, 1 \leq i \leq n$, be the points of local extrema of $\theta \mapsto\left|\Phi\left(e^{i \theta}\right)\right|$ on $(0, \pi), \varphi_{1}<\varphi_{2}<\cdots<\varphi_{n}$. Because of the symmetry we have $\varphi_{k}=k \pi /(n+1)$. Write $\varphi_{0}=0, \varphi_{n+1}=\pi$ and observe that between $\varphi_{k}$ and $\varphi_{k+1}, \theta \mapsto\left|\Phi\left(e^{i \theta}\right)\right|$ is either strictly increasing or strictly decreasing, $0 \leq k \leq n$; in particular, it is one-to-one. It is periodic with period $2 \pi /(n+1)$. Now it is easy to see that there is a function $F$ on $[a, b]$ such that $F\left(\left|\Phi\left(e^{i \varphi}\right)\right|\right)=\cos (n+1) \varphi$. By Theorem 4 the curve $\Gamma$ is singular. This completes the proof.

PROPOSITION 6. Every curve $\Gamma$ is the limit of a sequence of singular curves.

PROOF. We first prove that a curve $\Gamma$ is singular if the conformal map $\Phi$ is a polynomial. Let $\Phi(\varsigma)=\sum_{k=1}^{m} a_{k} \varsigma^{k}$. Since $\Phi(\bar{\zeta})=\overline{\Phi(\zeta)}(\varsigma \in \bar{\Delta}), a_{1}, \ldots, a_{m}$ are real. We have

$$
\begin{aligned}
\left|\Phi\left(e^{i \theta}\right)\right|^{2}= & \sum_{k=1}^{m} \sum_{l=1}^{m} a_{k} a_{l} e^{i(k-l) \theta} \\
= & a_{m} a_{1} e^{i(m-1) \theta}+\left(a_{m-1} a_{1}+a_{m} a_{2}\right) e^{i(m-2) \theta} \\
& +\cdots+\left(a_{2} a_{1}+a_{3} a_{2}+\cdots+a_{m} a_{m-1}\right) e^{i \theta} \\
& +\left(a_{1}^{2}+a_{2}^{2}+\cdots+a_{m}^{2}\right) \\
& +\left(a_{1} a_{2}+a_{2} a_{3}+\cdots+a_{m-1} a_{m}\right) e^{-i \theta} \\
& +\cdots+\left(a_{1} a_{m-1}+a_{2} a_{m}\right) e^{-i(m-2) \theta}+a_{1} a_{m} e^{-i(m-1) \theta}
\end{aligned}
$$

which shows that there are real numbers $A_{1}, \ldots, A_{m}$, not all equal to zero, such that $|\Phi(\varsigma)|^{2}=A_{1}(\varsigma+1 / \varsigma)+\cdots+A_{m}\left(\varsigma^{m}+1 / \varsigma^{m}\right)(\varsigma \in b \Delta)$. By Theorem 4 it follows that $\Gamma$ is singular.

Now, let $\Gamma$ be a curve and let $\Phi: \Delta \rightarrow D$ be the usual conformal map. Let $\Phi(\varsigma)=p_{1} \varsigma+p_{2} \varsigma^{2}+\cdots(\varsigma \in \Delta)$. Since $\Phi(\bar{\zeta})=\overline{\Phi(\varsigma)}(\varsigma \in \Delta), p_{k}$ are real, $k \in N$. Let $\varepsilon>0$. Since $\Phi$ is continuous on $\bar{\Delta}$ for each $n \in N$ there is an $r_{n}, 0<r_{n}<1$, such that

$$
\left|\Phi\left(r_{n} \zeta\right)-\Phi(\zeta)\right|<\varepsilon / n \quad(\varsigma \in b \Delta) .
$$

With no loss of generality assume that $r_{n} \nearrow 1$. Further, by the uniform convergence of the Taylor series on compact subsets of $\Delta$ it follows that for each $n \in N$ there is an $m \in N$ such that if $g_{n}(\varsigma)=p_{1} \varsigma+\cdots+p_{m} \zeta^{m}$ then $g_{n}$ maps $r_{n} \Delta$ conformally onto a domain $D_{n}$ bounded by a smooth curve $\Gamma_{n}$, and moreover that

$$
\left|g_{n}\left(r_{n} \zeta\right)-\Phi\left(r_{n} \zeta\right)\right|<\varepsilon / n \quad(\zeta \in b \Delta) .
$$


Since $p_{k}$ are real we have $\Gamma_{n}^{*}=\Gamma_{n}$. Further, if $\varepsilon$ is chosen small enough at the beginning then each $\Gamma_{n}$ contains 0 in its interior. Since $g_{n}$ are polynomials the curves $\Gamma_{n}$ are singular. By (6) and (7) it follows that $\Gamma$ is the limit of the curves $\Gamma_{n}$. This completes the proof.

Every curve $\Gamma$ is the limit of a sequence $\Gamma_{n}$ of the curves such that for each $n$, $\Gamma_{n}$ meets a circle centered at the origin in an arc. By Proposition 3 such curves are regular so we have

PROPOSITION 7. Every curve $\Gamma$ is the limit of a sequence of regular curves.

8. Functions analytic on circles. Let $\Gamma$ be a circle. We know from Example 2 in $\S 1$ that $\Gamma$ is singular and that the function $f(z)=1 / \bar{z}$ satisfies (1). Clearly $g(z)=z$ also satisfies (1) and so does every uniform limit on $\Omega$ of a sequence of polynomials in $f$ and $g$. It turns out that there are no other functions which satisfy (1).

THEOREM 5. Let $\Gamma$ be a circle. The $f \in C(\Omega)$ satisfies (1) if and only if $f$ is the uniform limit of a sequence of polynomials in $z$ and $1 / \bar{z}$. Consequently, if $f$ satisfies (1) then $f$ is analytic on every circle contained in $\Omega$ and containing 0 in its interior.

PROOF. The proof is similar to the proof of Theorem 3 but with $\Phi$ replaced by the linear map mapping $\Delta$ onto $D$ (thus dropping the requirement that $\Phi(0)=0$ ).

Let $\Gamma=\{\rho+\varsigma R: \varsigma \in b \Delta\}$ where $0<\rho<R$. Suppose that $f \in C(\Omega)$ satisfies (1). By Lemma 1 it follows that for each $n \in Z$ there is a function $G_{n}$, continuous on $\bar{\Delta}$, analytic in $\Delta$ such that $(\rho+\varsigma R)^{n} A_{n}(f,|\rho+\varsigma R|)=G_{n}(\varsigma)(\varsigma \in b \Delta)$. As in the proof of Theorem 3 we see that $A_{n}(r)=0(a \leq r \leq b, n<0)$. Let $n \geq 0$. We have $A_{n}(f,|\rho+\varsigma R|)=G_{n}(\varsigma) /(\rho+\varsigma R)^{n}(\varsigma \in b \Delta)$ which implies that

$$
G_{n}(\varsigma) /(\rho+\varsigma R)^{n}=G_{n}(\bar{\zeta}) /(\rho+\bar{\zeta} R)^{n}=\varsigma^{n} G_{n}(1 / \varsigma)(R+\varsigma \rho)^{n} \quad(\varsigma \in b \Delta)
$$

so

$$
\varsigma \mapsto \begin{cases}(R+\varsigma \rho)^{n} G_{n}(\varsigma) & (\varsigma \in \bar{\Delta}), \\ (\rho+\zeta R)^{n} \varsigma^{n} G_{n}(1 / \varsigma) & (\varsigma \in C \backslash \Delta),\end{cases}
$$

is a polynomial that we denote by $P_{n}$. We have $P_{n}(\varsigma)=\varsigma^{2 n} P_{n}(1 / \varsigma)(\varsigma \in C)$. Now,

$$
\begin{aligned}
A_{n}(f,|\rho+\varsigma R|) & =\frac{P_{n}(\varsigma)}{(\rho+\varsigma R)^{n}(R+\varsigma \rho)^{n}} \\
& =\frac{P_{n}(\varsigma) / \varsigma^{n}}{|\rho+\varsigma R|^{2 n}} \quad(\varsigma \in b \Delta),
\end{aligned}
$$

so there are $a_{0}, a_{1}, \ldots, a_{n}$ such that

$$
\left|\rho+R e^{i \varphi}\right|^{2 n} A_{n}\left(\left|\rho+R e^{i \varphi}\right|\right)=a_{0}+a_{1} \cos \varphi+\cdots+a_{n} \cos n \varphi .
$$

For every $m \in N, \cos m \varphi$ is a polynomial in $\cos \varphi$ of degree $m$, so

$$
\left|\rho+R e^{i \varphi}\right|^{2 n} A_{n}\left(\left|\rho+R e^{i \varphi}\right|\right)=Q_{n}(\cos \varphi)
$$

where $Q_{n}$ is a polynomial of degree $\leq n$. Write $\left|\rho+R e^{i \varphi}\right|=t$. Clearly $a \leq t \leq b$ $(0 \leq \varphi \leq \pi)$. We have $(\rho+R \cos \varphi)^{2}+R^{2} \sin ^{2} \varphi=t^{2}$ which implies that $\cos \varphi=$ $\left(t^{2}-R^{2}-\rho^{2}\right) / 2 \rho R$, so

$$
t^{2 n} A_{n}(t)=Q_{n}\left(\left(t^{2}-R^{2}-\rho^{2}\right) / 2 \rho R\right)=S_{n}\left(t^{2}\right)
$$


where $S_{n}$ is a polynomial of degree $\leq n$ so there are $c_{0}, c_{1}, \ldots, c_{n}$ such that

$$
A_{n}(r)=c_{0}+c_{1} / r^{2}+\cdots+c_{n} / r^{2 n} \quad(a \leq r \leq b) .
$$

If $z=r e^{i \varphi}$ it follows that

$$
\begin{aligned}
z^{n} A_{n}(|z|) & =z^{n}\left(c_{0}+c_{1} /(z \bar{z})+\cdots+c_{n} /\left(z^{n} \bar{z}^{n}\right)\right) \\
& =c_{0} z^{n}+c_{1} z^{n-1} / \bar{z}+\cdots+c_{n} / \bar{z}^{n} .
\end{aligned}
$$

Recall that on $\Omega, f(z)$ is the uniform limit of the sequence

$$
f_{m}(z)=m^{-1}\left(A_{0}(f,|z|)+\sum_{-1}^{1} A_{k}(f,|z|) z^{k}+\cdots+\sum_{-(m-1)}^{m-1} A_{k}(f,|z|) z^{k}\right)
$$

which implies that it is the uniform limit of a sequence of polynomials in $z$ and $1 / \bar{z}$. This completes the proof.

Thus, if $\Gamma$ is a circle then $f \in C(\Omega)$ satisfies (1) if and only if $f$ belongs to the function algebra on the closed annulus $\Omega$ generated by the functions $z$ and $1 / \bar{z}$. It might be interesting to study this algebra in the theory of function algebras.

ACKNOWLEDGment. The author is indebted to Franc Forstnerix and to Peter Gilkey for some stimulating discussions.

\section{REFERENCES}

1. J. Globevnik, Analyticity on rotation invariant families of curves, Trans. Amer. Math. Soc. $\mathbf{2 8 0}$ (1983), 247-254.

2. K. Hoffman, Banach spaces of analytic functions, Prentice-Hall, Englewood Cliffs, N. J., 1962.

3. W. Rudin, Real and complex analysis, McGraw-Hill, New York, 1966.

4. L. Zalcman, Offbeat integral geometry, Amer. Math. Monthly 87 (1980), 161-175.

Institute of Mathematics, Physics and Mechanics, E. K. University of LJUblJaNA, LJUblJaNA, YUgOSLAVia 